\title{
A SECOND ORDER ESTIMATE FOR COMPLEX HESSIAN EQUATIONS ON A COMPACT KÄHLER MANIFOLD
}

\author{
Zuoliang Hou, Xi-Nan Ma, and Damin Wu
}

\section{Introduction}

Let $(M, \omega)$ be an $n$-dimensional compact Kähler manifold. For a function $v$ on $M$, we denote

$$
\omega_{v}=\omega+d d^{c} v=\omega+\frac{\sqrt{-1}}{2 \pi} \partial \bar{\partial} v
$$

in which $d^{c}=\sqrt{-1}(\bar{\partial}-\partial) /(4 \pi)$. The complex Hessian equation on $M$ can be formulated as follows: Let $\sigma_{k}$ be the $k$-th elementary symmetric function, that is, for $1 \leq k \leq n$ and $\lambda=\left(\lambda_{1}, \ldots, \lambda_{n}\right) \in \mathbb{R}^{n}$,

$$
\sigma_{k}(\lambda)=\sum_{1 \leq i_{1}<\cdots<i_{k} \leq n} \lambda_{i_{1}} \cdots \lambda_{i_{k}} .
$$

Let $\lambda\left\{a_{i \bar{j}}\right\}$ denote the eigenvalues of Hermitian symmetric matrix $\left\{a_{i \bar{j}}\right\}$. We define

$$
\sigma_{k}\left(a_{i \bar{j}}\right)=\sigma_{k}\left(\lambda\left\{a_{i \bar{j}}\right\}\right) .
$$

The definition of $\sigma_{k}$ can be naturally extended to Kähler manifolds (and more generally, Hermitian manifolds). Let $A^{1,1}(M)$ be the space of smooth real $(1,1)$-forms on $M$; we define for each $\chi \in A^{1,1}(M)$,

$$
\sigma_{k}(\chi)=\left(\begin{array}{l}
n \\
k
\end{array}\right) \frac{\omega^{n-k} \wedge \chi^{k}}{\omega^{n}}
$$

For a smooth, positive function $f$ on $M$, the following equation is called the $k$-th complex Hessian equation on $M$ :

$$
\sigma_{k}\left(\omega_{u}\right)=\left(\begin{array}{l}
n \\
k
\end{array}\right) \frac{\omega^{n-k} \wedge \omega_{u}^{k}}{\omega^{n}}=f
$$

Throughout this paper, we fix $k$ to be an integer such that $2 \leq k \leq n-1$, unless otherwise indicated. Clearly, the compatibility condition for (1.2) is

$$
\int_{M} f \omega^{n}=\left(\begin{array}{l}
n \\
k
\end{array}\right) \int_{M} \omega^{n}
$$

It is well known that the famous Calabi conjecture in Kähler geometry is equivalent to the solvability of the equation (1.2) for $k=n$, which was solved by Yau [15] in 1976 . For the $k$-th Hessian equation, we consider the admissible solutions introduced by Caffarelli-Nirenberg-Spruck [2]:

Research of the second named author was supported by NSFC No. 10671186 and "Hundred People Program in Chinese Academy of Sciences". Part of this work was done while the second author was visiting the Institute for Advanced Study in 2008, where he was supported by National Science Foundation under No. DMS-0635607 and Zürich Financial Services. 
Definition. For $1 \leq k \leq n$, define

$$
\Gamma_{k}=\left\{\lambda \in \mathbb{R}^{n}: \sigma_{1}(\lambda)>0, \ldots, \sigma_{k}(\lambda)>0\right\} .
$$

Similarly, we define $\Gamma_{k}$ on $M$ as follows:

$$
\Gamma_{k}(M)=\left\{\chi \in A^{1,1}(M): \sigma_{j}(\chi)>0, j=1, \ldots, k\right\} .
$$

A function $u \in C^{2}(M)$ is called an admissible solution of (1.2) if $\omega_{u} \in \Gamma_{k}(M)$.

Note that the real counterparts of (1.2) have been studied extensively in the literature, as these equations appear naturally and play important roles in classical geometry and conformal geometry. For example, the Dirichlet problem of a Hessian equation in $\mathbb{R}^{n}$ was studied by Caffarelli-Nirenberg-Spruck [2] and Ivochkina [9]. Pogorelov [12], Guan-Ma [6] investigated the Christoffel-Minkowski problem, which is related to the convex solution of a class Hessian equation. The existence of starshaped hypersurfaces with prescribed Weingarten curvature was studied by CaffarelliNirenberg-Spruck [3]. For Hessian equations in conformal geometry, see for example, Chang-Gursky-Yang [4] and Guan-Wang [7]. The Hessian equations on Riemannian manifolds were studied by Y. Y. Li [11] and Urbas [13]. For more details, we refer to $\mathrm{X}$. J. Wang [14] and the references therein.

The complex Hessian equation, however, is much less studied so far. As we know, the Dirichlet problem of complex Hessian equation in $\mathbb{C}^{n}$ was studied by S. Y. Li [10] and Błocki [1]. The first named author [8] proved the uniqueness of the solution of (1.2); he [8] also proved the existence of a smooth admissible solution of (1.2), by assuming the nonnegativity of the orthogonal bisectional curvature of $\omega$.

A natural approach to solve (1.2) is the continuity method. The openness follows from the implicit function theory. The closedness argument depends on the a priori estimates up to the second order, in view of the standard Evans-Krylov theory. Similar to the Monge-Ampère equation, the zero order estimate follows from Yau's Moser iteration [15]. In this paper, we establish a second order estimate for (1.2).

Throughout this paper, we denote by $C_{0}$ a nonnegative constant depending only on the lower bound of the bisectional curvature of $\omega$. More precisely, for each point $x$ in $M$, for any two unit vectors $\eta$ and $\zeta$ of $T_{x}^{1,0} M$, we denote

$$
R_{\eta \bar{\eta} \zeta \bar{\zeta}}(x)=R(\eta, \bar{\eta}, \zeta, \bar{\zeta})(x) ;
$$

then we set

$$
C_{0}=\sup _{x \in M}\left|\inf _{\eta, \zeta}\left(R_{\eta \bar{\eta} \zeta \bar{\zeta}}\right)(x)\right|,
$$

in which the infimum runs through all two unit vectors $\eta, \zeta$ in $T_{x}^{1,0} M$ for a point $x$ in $M$. Our theorem is as follows:

Theorem 1.1. Let $u \in C^{4}(M)$ be an admissible solution of equation (1.2) and $f$ satisfy the compatibility condition (1.3). Let $C_{0}$ be the constant defined by (1.4). Then, under the normalized condition

$$
\int_{M} u \omega^{n}=0
$$

we have the following uniform second derivative estimate

$$
\sup \left|d d^{c} u\right|_{\omega} \leq C\left(\sup _{M}|\nabla u|_{\omega}^{2}+1\right),
$$


where $C$ is a positive constant depending only on $\left\|f^{\frac{1}{k}}\right\|_{C^{2}(M)}, \omega$, and $C_{0}, n, k$.

We remark that the $(1,1)$-form $\omega_{u}$ in (1.2) allows negative eigenvalues. This feature breaks down the classic methods for Monge-Amperè type equations, as they require at least semipositivity of $\omega_{u}$. Here we adopt a casewise argument, motivated by the treatment of Chou-Wang [5] on the real Hessian equation. The complex case is however more subtle in handling the third order terms, due to the difference between $\left|u_{1 \overline{1} \bar{i}}\right|^{2}$ and $\left|u_{11 \bar{i}}\right|^{2}$. The difference remains to be the major difficulty in deriving a Pogorelov type interior $C^{2}$ estimate for the complex Monge-Ampère equation on a bounded domain in $\mathbb{C}^{n}$. We overcome this difficulty here by squeezing out a small positivity in the third order terms and absorbing the negative effect via the Kähler form.

Moreover, we improve the estimate in [5] so that the constant $C$ in (1.5) does not depend on $\inf _{M} f$ (see Lemma 2.2 part (2)). This means that our estimate still holds for the degenerate case. Let us finally point out that an estimate of the form (1.5) is also adapted to the blowing up analysis.

\section{Estimates}

First, we remark that the following zeroth order estimate follows directly from Yau's Moser iteration method. Its proof is therefore omitted.

Lemma 2.1. If $u \in C^{2}(M)$ is an admissible solution of (1.2) and $f$ satisfies the compatibility condition (1.3), then under the normalization condition

$$
\int_{M} u \omega^{n}=0
$$

we have the following uniform estimates

$$
\sup _{M}|u| \leq C
$$

where $C$ is a positive constant depending only on $\sup _{M}|f|$ and $\omega$.

To show Theorem 1.1, our calculations will be carried out at a point $x$ in the manifold $M$. Let $\left(z_{1}, \ldots, z_{n}\right)$ be a local holomorphic coordinate system centered at $x$. The subscripts of a function $h$ always denote the covariant derivatives of $h$ with respect to $\omega$ in the directions of the local frame $\left(\partial / \partial z^{1}, \ldots, \partial / \partial z^{n}\right)$. Namely,

$$
h_{i}=\nabla_{\partial / \partial z^{i}} h, \quad h_{i \bar{j}}=\nabla_{\partial / \partial \bar{z}^{j}} \nabla_{\partial / \partial z^{i}} h, \quad h_{i \bar{j} l}=\nabla_{\partial / \partial z^{l}} \nabla_{\partial / \partial \bar{z}^{j}} \nabla_{\partial / \partial z^{i}} h .
$$

Proof of Theorem 1.1. Let us consider the function

$$
W(x, \xi)=\left(1+u_{i \bar{j}} \xi^{i} \bar{\xi}^{j}\right) \exp \left[\varphi\left(|\nabla u|^{2}\right)+\psi(u)\right],
$$

for any $x$ in $M$ and any unit vector $\xi \in T_{x}^{1,0} M$. Here

$$
\varphi(t)=-\frac{1}{2} \log \left(1-\frac{t}{2 K}\right)
$$

in which

$$
K=\sup _{M}|\nabla u|^{2}+1
$$


and

$$
\psi(t)=-A \log \left(1+\frac{t}{2 L}\right)
$$

in which

$$
L=\sup _{M}|u|+1, \quad A=3 L\left(2 C_{0}+1\right) .
$$

By (2.3) we have

$$
\begin{aligned}
\frac{1}{2} \log 2 & \geq \varphi\left(|\nabla u|^{2}\right) \geq 0, \\
\frac{1}{2 K} & \geq \varphi^{\prime}\left(|\nabla u|^{2}\right) \geq \frac{1}{4 K}>0, \\
\varphi^{\prime \prime}\left(|\nabla u|^{2}\right) & =2\left[\varphi^{\prime}\left(|\nabla u|^{2}\right)\right]^{2}>0 .
\end{aligned}
$$

It follows from (2.5) that

$$
\begin{aligned}
A \log 2 & \geq \psi \geq A \log (2 / 3), \\
\frac{A}{L} & \geq-\psi^{\prime} \geq \frac{A}{3 L}=2 C_{0}+1, \\
\psi^{\prime \prime} & \geq \frac{2 \epsilon}{1-\epsilon}\left(\psi^{\prime}\right)^{2}, \quad \text { for all } \epsilon \leq \frac{1}{2 A+1} .
\end{aligned}
$$

These inequalities will be used below.

Since $M$ is compact, $W$ attains its maximum at some point $x_{0}$ in a unit tangent direction $\xi_{0} \in T_{x_{0}}^{1,0} M$. Since $M$ is Kähler, we can choose a local normal coordinate system near $x_{0}$ such that

$$
g_{i \bar{j}}\left(x_{0}\right)=\delta_{i j}, \quad \frac{\partial g_{i \bar{j}}}{\partial z^{l}}\left(x_{0}\right)=0, \quad u_{i \bar{j}}\left(x_{0}\right)=\delta_{i j} u_{i \bar{i}}\left(x_{0}\right) .
$$

In addition, we may assume that

$$
\lambda_{1} \geq \lambda_{2} \geq \cdots \geq \lambda_{n}
$$

Here and in what follows, we denote by

$$
\lambda_{i}=\left(1+u_{i \bar{i}}\right)\left(x_{0}\right), \quad \text { for all } i=1, \ldots, n .
$$

Then, by definition of $W$ and the above construction, we know that $\xi_{0}$ must be coincide with $\partial / \partial z^{1}$ at $x_{0}$. We can extend $\xi_{0}$ to a smooth unit vector field in the neighborhood of $x_{0}$ by setting

$$
\xi_{0}=g_{1 \overline{1}}^{-1 / 2} \frac{\partial}{\partial z^{1}} .
$$

In the follows, the covariant derivatives with respect to $\omega$ are given in terms of this local coordinate system, unless otherwise indicated.

Our goal is to derive a uniform upper bound for $\lambda_{1}$. Without loss of generality, we can assume that $\lambda_{1} \geq 1$.

Note that the function

$$
G(x)=\log W\left(x, \xi_{0}\right)=\log \left(1+g_{1 \overline{1}}^{-1} u_{1 \overline{1}}\right)+\varphi\left(|\nabla u|^{2}\right)+\psi(u)
$$


is well-defined in a neighborhood of $x_{0}$, and $G$ achieves its maximum at $x_{0}$. From now on, all the calculations will be carried out at the point $x_{0}$. (Let us bear in mind that covariant differentiating the metric tensor always equals zero.) First, we have

$$
0=G_{i}=\frac{u_{1 \overline{1} i}}{1+u_{1 \overline{1}}}+\varphi^{\prime} u_{i} u_{\bar{i} i}+\varphi^{\prime} \sum_{p=1}^{n} u_{p i} u_{\bar{p}}+\psi^{\prime} u_{i},
$$

for each $i=1, \ldots, n$. Note that

$$
\begin{aligned}
G_{i \bar{j}} & =\frac{u_{1 \overline{1} i \bar{j}}}{1+u_{1 \overline{1}}}-\frac{u_{1 \bar{i} i} u_{1 \overline{1} \bar{j}}}{\left(1+u_{1 \overline{1}}\right)^{2}}+\psi^{\prime} u_{i \bar{j}}+\psi^{\prime \prime} u_{i} u_{\bar{j}} \\
& +\varphi^{\prime \prime}\left(u_{i} u_{i \bar{i}}+\sum_{p=1}^{n} u_{p i} u_{\bar{p}}\right)\left(u_{\bar{j}} u_{j \bar{j}}+\sum_{q=1}^{n} u_{q} u_{\bar{q} \bar{j}}\right) \\
& +\varphi^{\prime}\left(\delta_{i j} u_{i \bar{i}}^{2}+\sum_{p=1}^{n} u_{p i} u_{\bar{p} \bar{j}}\right)+\varphi^{\prime} \sum_{p=1}^{n}\left(u_{p i \bar{j}} u_{\bar{p}}+u_{\bar{p} i \bar{j}} u_{p}\right) .
\end{aligned}
$$

Let

$$
F\left(\omega_{u}\right)=\left(\sigma_{k}\left(\omega_{u}\right)\right)^{1 / k}
$$

We denote by

$$
F^{i \bar{j}}=\frac{\partial F}{\partial w_{i \bar{j}}}, \quad F^{i \bar{j}, p \bar{q}}=\frac{\partial^{2} F}{\partial w_{i \bar{j}} \partial w_{p \bar{q}}},
$$

where $\left(w_{u}\right)_{i \bar{j}}=g_{i \bar{j}}+u_{i \bar{j}}$. Then, the positive definite matrix $\left(F^{i \bar{j}}\left(\omega_{u}\right)\right)$ is diagonalized at the point $x_{0}$. More precisely, we have

$$
F^{i \bar{j}}\left(\omega_{u}\right)=\delta_{i j} F^{i \bar{i}}\left(\omega_{u}\right)=\frac{1}{k}\left[\sigma_{k}(\lambda)\right]^{1 / k-1} \sigma_{k-1}(\lambda \mid i) \delta_{i j} .
$$

Furthermore, at $x_{0}$,

$$
F^{i \bar{j}, p \bar{q}}\left(\omega_{u}\right)= \begin{cases}F^{i \bar{i}, p \bar{p}}, & \text { if } i=j, p=q ; \\ F^{i \bar{p}, p \bar{i}}, & \text { if } i=q, p=j, i \neq p ; \\ 0, & \text { otherwise, }\end{cases}
$$

in which

$$
\begin{aligned}
F^{i \bar{i}, p \bar{p}}= & \frac{1}{k}\left[\sigma_{k}(\lambda)\right]^{1 / k-1}\left(1-\delta_{i p}\right) \sigma_{k-2}(\lambda \mid i p) \\
& +\frac{1}{k}\left(\frac{1}{k}-1\right)\left[\sigma_{k}(\lambda)\right]^{1 / k-2} \sigma_{k-1}(\lambda \mid i) \sigma_{k-1}(\lambda \mid p), \\
F^{i \bar{p}, \bar{p} \bar{i}}= & -\frac{1}{k}\left[\sigma_{k}(\lambda)\right]^{1 / k-1} \sigma_{k-2}(\lambda \mid i p) .
\end{aligned}
$$

Here and in the follows, $\sigma_{r}\left(\lambda \mid i_{1} \ldots i_{l}\right)$, with $i_{1}, \ldots, i_{l}$ being distinct, stands for the $r$-th symmetric function with $\lambda_{i_{1}}=\cdots=\lambda_{i_{l}}=0$. We have, in addition,

$$
\sum_{i=1}^{n} F^{i \bar{i}} w_{i \bar{i}}=\sum_{i=1}^{n} F^{i \bar{i}} \lambda_{i}=\sigma_{k}^{1 / k}=f^{1 / k}
$$


Thus, at the point $x_{0}$,

$$
\begin{aligned}
0 & \geq \sum_{i, j=1}^{n} F^{i \bar{j}} G_{i \bar{j}}=\sum_{i=1}^{n} F^{i \bar{i}} G_{i \bar{i}} \\
& =\sum_{i=1}^{n} \frac{F^{i \bar{i}} u_{1 \overline{1} i \bar{i}}}{1+u_{1 \overline{1}}}-\sum_{i=1}^{n} \frac{F^{i \bar{i}}\left|u_{1 \overline{1} i}\right|^{2}}{\left(1+u_{1 \overline{1}}\right)^{2}}+\psi^{\prime} \sum_{i=1}^{n} F^{i \bar{i}} u_{i \bar{i}} \\
& +\psi^{\prime \prime} \sum_{i=1}^{n} F^{i \bar{i}}\left|u_{i}\right|^{2}+\varphi^{\prime \prime} \sum_{i=1}^{n} F^{i \bar{i}}\left|u_{i} u_{i \bar{i}}+\sum_{p=1}^{n} u_{p i} u_{\bar{p}}\right|^{2} \\
& +\varphi^{\prime} \sum_{i=1}^{n} F^{i \bar{i}} u_{i \bar{i}}^{2}+\varphi^{\prime} \sum_{i, p=1}^{n} F^{i \bar{i}}\left|u_{p i}\right|^{2}+\varphi^{\prime} \sum_{i, p=1}^{n} F^{i \bar{i}}\left(u_{p i \bar{i}} u_{\bar{p}}+u_{\bar{p} i \bar{i}} u_{p}\right) .
\end{aligned}
$$

For an arbitrary smooth function $h$ on $M$, covariant differentiating the equation

$$
F\left(\omega_{u}\right)=h
$$

in the $\partial / \partial z^{l}$ direction yields that

$$
F^{i \bar{j}} u_{i \bar{j} l}=h_{l}
$$

Here the summation convention is used. Differentiating again in the $\partial / \partial \bar{z}^{m}$ direction, we obtain that

$$
F^{i \bar{j}} u_{i \bar{j} l \bar{m}}+F^{i \bar{j}, p \bar{q}} u_{i \bar{j} l} u_{p \bar{q} \bar{m}}=h_{l \bar{m}} .
$$

By commuting the covariant derivatives, we have

$$
\begin{aligned}
u_{i \bar{j} l} & =u_{i l \bar{j}}-u_{a} R_{i l \bar{j}}^{a} \\
u_{i \bar{j} l \bar{m}} & =u_{l \bar{m} i \bar{j}}+u_{a \bar{j}} R_{i l \bar{m}}^{a}-u_{b \bar{m}} R_{i l \bar{j}}^{b} .
\end{aligned}
$$

This implies that

$$
\begin{aligned}
F^{i \bar{j}} u_{l i \bar{j}} & =h_{l}+u_{a} F^{i \bar{j}} R_{l i \bar{j}}^{a}, \\
F^{i \bar{j}} u_{l \bar{m} i \bar{j}} & =h_{l \bar{m}}-F^{i \bar{j}, p \bar{q}} u_{i \bar{j} l} u_{p \bar{q} \bar{m}}+F^{i \bar{j}} u_{b \bar{m}} R_{l i \bar{j}}^{b}-F^{i \bar{j}} u_{a \bar{j}} R_{i l \bar{m}}^{a} .
\end{aligned}
$$

In particular, at the point $x_{0}$, we have for $h=f^{1 / k}$,

$$
\begin{aligned}
\sum_{i}^{n} F^{i \bar{i}} u_{p i \bar{i}} & =\left(f^{1 / k}\right)_{p}+\sum_{i, q=1}^{n} u_{q} F^{i \bar{i}} R_{p \bar{q} \bar{i}} \\
\sum_{i=1}^{n} F^{i \bar{i}} u_{1 \overline{1} i \bar{i}} & =\left(f^{1 / k}\right)_{1 \overline{1}}-\sum_{i, j, p, q=1}^{n} F^{i \bar{j}, p \bar{q}} u_{i \bar{j} 1} u_{p \bar{q} \overline{1}}+\sum_{i=1}^{n} F^{i \bar{i}}\left(u_{1 \overline{1}}-u_{i \bar{i}}\right) R_{1 \overline{1} i \bar{i}}
\end{aligned}
$$

Recall that

$$
1+u_{i \bar{i}}\left(x_{0}\right)=w_{i \bar{i}}\left(x_{0}\right)=\lambda_{i}
$$


for each $i=1, \ldots, n$. We have

$$
\begin{aligned}
\sum_{i=1}^{n} F^{i \bar{i}}\left(u_{1 \overline{1}}-u_{i \bar{i}}\right) R_{1 \overline{1} i \bar{i}} & =\sum_{i=2}^{n} F^{i \bar{i}}\left(\lambda_{1}-\lambda_{i}\right) R_{1 \overline{1} i \bar{i}} \\
& \geq \inf _{i \neq l}\left(R_{\bar{i} \bar{i} \bar{l}}\right) \sum_{i=2}^{n} F^{i \bar{i}}\left(\lambda_{1}-\lambda_{i}\right) \\
& =\inf _{i \neq l}\left(R_{i \bar{i} i \bar{l}}\right)\left(\lambda_{1} \sum_{i=1}^{n} F^{i \bar{i}}-f^{1 / k}\right), \quad(\text { by }(2.17)) .
\end{aligned}
$$

For convenience, we denote by

$$
\mathcal{F}=\sum_{i=1}^{n} F^{i \bar{i}}\left(x_{0}\right) .
$$

Thus, by (2.20),

$$
\begin{aligned}
\sum_{i=1}^{n} \frac{F^{i \bar{i}} u_{1 \overline{1} i \bar{i}}}{1+u_{1 \overline{1}}} & \geq-\lambda_{1}^{-1} \sum_{i, j, p, q=1}^{n} F^{i \bar{j}, p \bar{q}} u_{i \bar{j} 1} u_{p \bar{q} \overline{1}}-C_{0} \mathcal{F} \\
& -C_{0} \sup _{M} f^{1 / k}-\sup _{M}\left|d d^{c}\left(f^{1 / k}\right)\right|_{\omega} .
\end{aligned}
$$

Here $C_{0}$ is the constant given by (1.4).

It follows from (2.19) that

$$
\begin{aligned}
& \varphi^{\prime} \sum_{i, p=1}^{n} F^{i \bar{i}}\left(u_{p i \bar{i}} u_{\bar{p}}+u_{\bar{p} i \bar{i}} u_{p}\right) \\
& =\varphi^{\prime} \sum_{p=1}^{n}\left[\left(f^{1 / k}\right)_{p} u_{\bar{p}}+\left(f^{1 / k}\right)_{\bar{p}} u_{p}\right]+\varphi^{\prime} \sum_{i, p, q=1}^{n} u_{\bar{p}} u_{q} F^{i \bar{i}} R_{i \bar{i} p \bar{q}} \\
& \geq-\varphi^{\prime}\left|\nabla\left(f^{1 / k}\right)\right|^{2}-\varphi^{\prime}|\nabla u|^{2}-C_{0} \varphi^{\prime}|\nabla u|^{2} \mathcal{F} \\
& \geq-\frac{\sup _{M}\left|\nabla\left(f^{1 / k}\right)\right|^{2}}{2}-\frac{1}{2}-\frac{C_{0}}{2} \mathcal{F}, \quad(\text { by }(2.8)) .
\end{aligned}
$$

Moreover, apply (2.17) to obtain that

$$
\begin{aligned}
\psi^{\prime} \sum_{i=1}^{n} F^{i \bar{i}} u_{i \bar{i}} & =\psi^{\prime} \sum_{i=1}^{n} F^{i \bar{i}}\left(\lambda_{i}-1\right)=\psi^{\prime} f^{1 / k}-\psi^{\prime} \mathcal{F} \\
& \geq-3\left(2 C_{0}+1\right) \sup _{M} f^{1 / k}-\psi^{\prime} \mathcal{F}, \quad(\text { by }(2.11)) .
\end{aligned}
$$


Similarly, we have

$$
\begin{aligned}
\varphi^{\prime} \sum_{i=1}^{n} F^{i \bar{i}} u_{i \bar{i}}^{2} & =\varphi^{\prime} \sum_{i=1}^{n} F^{i \bar{i}}\left(\lambda_{i}-1\right)^{2} \\
& =\varphi^{\prime} \sum_{i=1}^{n} F^{i \bar{i}} \lambda_{i}^{2}-2 \varphi^{\prime} f^{1 / k}+\varphi^{\prime} \mathcal{F} \\
& \geq \varphi^{\prime} \sum_{i=1}^{n} F^{i \bar{i}} \lambda_{i}^{2}-\sup _{M} f^{1 / k}+\varphi^{\prime} \mathcal{F}
\end{aligned}
$$

Substituting (2.21), (2.22), (2.23), (2.24) into (2.18) yields that

$$
\begin{aligned}
0 & \geq-\sum_{i, j, p, q=1}^{n} \frac{F^{i \bar{j}, p \bar{q}} u_{i \bar{j} 1} u_{p \bar{q} \overline{1}}}{1+u_{1 \overline{1}}}-\sum_{i=1}^{n} \frac{F^{i \bar{i}}\left|u_{1 \overline{1} i}\right|^{2}}{\left(1+u_{1 \overline{1}}\right)^{2}}+\psi^{\prime \prime} \sum_{i=1}^{n} F^{i \bar{i}}\left|u_{i}\right|^{2} \\
& +\varphi^{\prime \prime} \sum_{i=1}^{n} F^{i \bar{i}}\left|u_{i} u_{i \bar{i}}+\sum_{p=1}^{n} u_{p i} u_{\bar{p}}\right|^{2}+\varphi^{\prime} \sum_{i=1}^{n} F^{i \bar{i}} \lambda_{i}^{2}+\varphi^{\prime} \sum_{i, p=1}^{n} F^{i \bar{i}}\left|u_{p i}\right|^{2} \\
& +\left(-\psi^{\prime}+\varphi^{\prime}-2 C_{0}\right) \mathcal{F}-C_{2},
\end{aligned}
$$

where $C_{2}$ is a positive constant depending only on $C_{0}, \sup f^{1 / k}, \sup \left|\nabla\left(f^{1 / k}\right)\right|^{2}$, and $\sup \left|d d^{c}\left(f^{1 / k}\right)\right|$. From here we would derive the estimates separately for two cases, either $\lambda_{n}<-\delta \lambda_{1}$, or $\lambda_{n} \geq-\delta \lambda_{1}$. Here and below, we set

$$
\delta=\frac{1}{1+2 A}=\left[1+6\left(\sup _{M}|u|+1\right)\left(2 C_{0}+1\right)\right]^{-1}>0
$$

in which $A$ is the constant given by (2.6).

Let us first consider Case 1: Suppose that $\lambda_{n}<-\delta \lambda_{1}$. We apply (2.14) to obtain that

$$
\begin{aligned}
-\sum_{i=1}^{n} \frac{F^{i \bar{i}}\left|u_{1 \bar{i} i}\right|^{2}}{\left(1+u_{1 \overline{1}}\right)^{2}} & =-\sum_{i=1}^{n} F^{i \bar{i}}\left|\varphi^{\prime}\left(u_{i} u_{i \bar{i}}+\sum_{p=1}^{n} u_{p i} u_{\bar{p}}\right)+\psi^{\prime} u_{i}\right|^{2} \\
& \geq-2\left(\varphi^{\prime}\right)^{2} \sum_{i=1}^{n} F^{i \bar{i}}\left|u_{i} u_{i \bar{i}}+\sum_{p=1}^{n} u_{p i} u_{\bar{p}}\right|^{2}-2\left(\psi^{\prime}\right)^{2}|\nabla u|^{2} \mathcal{F} \\
& \geq-2\left(\varphi^{\prime}\right)^{2} \sum_{i=1}^{n} F^{i \bar{i}}\left|u_{i} u_{i \bar{i}}+\sum_{p=1}^{n} u_{p i} u_{\bar{p}}\right|^{2}-2\left(6 C_{0}+3\right)^{2} K \mathcal{F}, \quad(\text { by }(2.11)) .
\end{aligned}
$$

On the right hand side of (2.25), the first term can be dropped, in view of the concavity of $F$; so does the seventh term, since by (2.8) and (2.11),

$$
-\psi^{\prime}+\varphi^{\prime}-2 C_{0} \geq-\psi^{\prime}-2 C_{0} \geq 1 \text {. }
$$

It follows that

$$
\begin{aligned}
0 \geq & {\left[\varphi^{\prime \prime}-2\left(\varphi^{\prime}\right)^{2}\right] \sum_{i=1}^{n} F^{i \bar{i}}\left|u_{i} u_{i \bar{i}}+\sum_{p=1}^{n} u_{p i} u_{\bar{p}}\right|^{2} } \\
& +\varphi^{\prime} \sum_{i=1}^{n} F^{i \bar{i}} \lambda_{i}^{2}-18\left(2 C_{0}+1\right)^{2} K \mathcal{F}-C_{2}
\end{aligned}
$$


Applying (2.8) and (2.9) to (2.26) yields that

$$
0 \geq \frac{1}{4 K} F^{n \bar{n}} \lambda_{n}^{2}-18\left(2 C_{0}+1\right)^{2} K \mathcal{F}-C_{2} .
$$

Note that

$$
F^{n \bar{n}} \geq \frac{\mathcal{F}}{n}
$$

This is due to the monotonicity $F^{n \bar{n}} \geq \cdots \geq F^{1 \overline{1}}$, which, in turn, follows from (2.15) and that

$$
\sigma_{k-1}(\lambda \mid i)-\sigma_{k-1}(\lambda \mid j)=\left(\lambda_{j}-\lambda_{i}\right) \sigma_{k-2}(\lambda \mid i j) \geq 0, \quad \text { for any } i>j .
$$

Substituting (2.28) into (2.27) yields that

$$
0 \geq \frac{\mathcal{F}}{4 K n} \lambda_{n}^{2}-18\left(2 C_{0}+1\right)^{2} K \mathcal{F}-C_{2} .
$$

By the assumption of Case $1, \lambda_{n}<-\delta \lambda_{1}$, we obtain that

$$
\lambda_{1}^{2} \leq \frac{72 n\left(2 C_{0}+1\right)^{2} K^{2}}{\delta^{2}}+\frac{4 n K C_{2}}{\delta^{2} \mathcal{F}} .
$$

To finish Case 1, we note that $\mathcal{F}$ has a uniform lower bound. Indeed,

$$
\begin{aligned}
\mathcal{F} & =\sum_{i=1}^{n} F^{i \bar{i}}=\frac{1}{k}\left[\sigma_{k}(\lambda)\right]^{1 / k-1} \sum_{i=1}^{n} \sigma_{k-1}(\lambda \mid i) \quad(\text { by }(2.15)) \\
& =\frac{n-k+1}{k}\left[\sigma_{k}(\lambda)\right]^{1 / k-1} \sigma_{k-1}(\lambda) \geq 1, \quad \text { (by Maclaurin's inequality). }
\end{aligned}
$$

Therefore, we conclude that

$$
\lambda_{1} \leq \frac{\left[72 n\left(2 C_{0}+1\right)^{2}+4 n C_{2}\right]^{1 / 2}}{\delta} K=C K
$$

where $C>0$ is a constant depending only on $C_{0}, n, \sup |u|, \sup f^{1 / k}, \sup \left|\nabla\left(f^{1 / k}\right)\right|^{2}$, and $\sup \left|d d^{c}\left(f^{1 / k}\right)\right|$.

Now let us consider Case 2: We assume that $\lambda_{n} \geq-\delta \lambda_{1}$. Recall that

$$
\delta=\frac{1}{1+2 A}=\left[1+6\left(\sup _{M}|u|+1\right)\left(2 C_{0}+1\right)\right]^{-1}>0 .
$$

Let

$$
I=\left\{i \in\{1, \ldots, n\} \mid \sigma_{k-1}(\lambda \mid i)>\delta^{-1} \sigma_{k-1}(\lambda \mid 1)\right\} .
$$

Clearly, $1 \notin I$, since $\delta^{-1} \geq 7$. By (2.15) we know that an index $j$ is contained in $I$ if and only if

$$
F^{j \bar{j}}\left(x_{0}\right)>\delta^{-1} F^{1 \overline{1}}\left(x_{0}\right) .
$$

Let us first treat those indices which are not in $I$ : By (2.14) we obtain that

$$
\begin{aligned}
-\sum_{i \notin I} \frac{F^{i \bar{i}}\left|u_{1 \bar{i}}\right|^{2}}{\left(1+u_{1 \overline{1}}\right)^{2}} & \geq-2\left(\varphi^{\prime}\right)^{2} \sum_{i \notin I} F^{i \bar{i}}\left|u_{i} u_{i \bar{i}}+\sum_{p=1}^{n} u_{p i} u_{\bar{p}}\right|^{2}-2\left(\psi^{\prime}\right)^{2} \sum_{i \notin I} F^{i \bar{i}}\left|u_{i}\right|^{2} \\
& \geq-2\left(\varphi^{\prime}\right)^{2} \sum_{i \notin I} F^{i \bar{i}}\left|u_{i} u_{i \bar{i}}+\sum_{p=1}^{n} u_{p i} u_{\bar{p}}\right|^{2}-18\left(2 C_{0}+1\right)^{2} K F^{1 \overline{1}} .
\end{aligned}
$$


The first term on the right can be absorbed by the term

$$
\varphi^{\prime \prime} \sum_{i \notin I} F^{i \bar{i}}\left|u_{i} u_{i \bar{i}}+\sum_{p=1}^{n} u_{p i} u_{\bar{p}}\right|^{2},
$$

in view of (2.9). It then follows from (2.25) and (2.8) that

$$
\begin{aligned}
0 & \geq-\sum_{i, j, p, q=1}^{n} \frac{F^{i \bar{j}, p \bar{q}} u_{i \bar{j} 1} u_{p \bar{q} \overline{1}}}{1+u_{1 \overline{1}}}-\sum_{i \in I} \frac{F^{i \bar{i}}\left|u_{1 \overline{1} i}\right|^{2}}{\left(1+u_{1 \overline{1}}\right)^{2}}+\left(-\psi^{\prime}+\varphi^{\prime}-2 C_{0}\right) \mathcal{F} \\
& +\varphi^{\prime \prime} \sum_{i \in I} F^{i \bar{i}}\left|u_{i} u_{i \bar{i}}+\sum_{p=1}^{n} u_{p i} u_{\bar{p}}\right|^{2}+\psi^{\prime \prime} \sum_{i=1}^{n} F^{i \bar{i}}\left|u_{i}\right|^{2} \\
& +\frac{1}{4 K} \sum_{i=1}^{n} F^{i \bar{i}} \lambda_{i}^{2}-18\left(2 C_{0}+1\right)^{2} K F^{1 \overline{1}}-C_{2} .
\end{aligned}
$$

Without loss of generality, we can assume that

$$
\lambda_{1}^{2} \geq\left[12\left(2 C_{0}+1\right) K\right]^{2},
$$

for, otherwise the desired bound is obtained by

$$
\lambda_{1} \leq 12\left(2 C_{0}+1\right) K
$$

Thus, we have

$$
\begin{aligned}
0 & \geq-\sum_{i, j, p, q=1}^{n} \frac{F^{i \bar{j}, p \bar{q}} u_{i \bar{j} 1} u_{p \bar{q} \overline{1}}}{1+u_{1 \overline{1}}}-\sum_{i \in I} \frac{F^{i \bar{i}}\left|u_{1 \overline{1} i}\right|^{2}}{\left(1+u_{1 \overline{1}}\right)^{2}} \\
& +\psi^{\prime \prime} \sum_{i=1}^{n} F^{i \bar{i}}\left|u_{i}\right|^{2}+\varphi^{\prime \prime} \sum_{i \in I} F^{i \bar{i}}\left|u_{i} u_{i \bar{i}}+\sum_{p=1}^{n} u_{p i} u_{\bar{p}}\right|^{2} \\
& +\frac{1}{8 K} \sum_{i=1}^{n} F^{i \bar{i}} \lambda_{i}^{2}+\left(-\psi^{\prime}-2 C_{0}\right) \mathcal{F}-C_{2} .
\end{aligned}
$$

We claim that the following estimate holds:

$$
\begin{aligned}
& -\sum_{i, j, p, q=1}^{n} \frac{F^{i \bar{j}, p \bar{q}} u_{i \bar{j} 1} u_{p \bar{q} \overline{1}}}{1+u_{1 \overline{1}}}-\sum_{i \in I} \frac{F^{i \bar{i}}\left|u_{1 \overline{1} i}\right|^{2}}{\left(1+u_{1 \overline{1}}\right)^{2}}+\varphi^{\prime \prime} \sum_{i \in I} F^{i \bar{i}}\left|u_{i} u_{i \bar{i}}+\sum_{p=1}^{n} u_{p i} u_{\bar{p}}\right|^{2} \\
& +\psi^{\prime \prime} \sum_{i=1}^{n} F^{i \bar{i}}\left|u_{i}\right|^{2} \geq 0 .
\end{aligned}
$$

Assuming this claim, we obtain from (2.29) that

$$
\begin{aligned}
C_{2} & \geq\left(-\psi^{\prime}-2 C_{0}\right) \mathcal{F}+\frac{1}{8 K} \sum_{i=1}^{n} F^{i \bar{i}} \lambda_{i}^{2} \\
& \geq \mathcal{F}+\frac{1}{8 K} F^{1 \overline{1}} \lambda_{1}^{2}, \quad \text { by }(2.11) .
\end{aligned}
$$

This in particular implies that $\mathcal{F} \leq C_{2}$. By the following Lemma 2.2 part (2) we obtain that

$$
F^{1 \overline{1}} \geq \frac{c(n, k)}{C_{2}^{k-1}}
$$


where $c(n, k)$ is a positive constant depending only on $n$ and $k$. Substituting this back into (2.31) yields the desired bound

$$
\lambda_{1} \leq \frac{2 \sqrt{2} C_{2}^{k / 2}}{c(n, k)^{1 / 2}} \sqrt{K} .
$$

Here we recall that $C_{2}>0$ is a constant depending only $C_{0}, \sup f^{1 / k}, \sup \left|\nabla\left(f^{1 / k}\right)\right|^{2}$, and $\sup \left|d d^{c} f\right|$, as given in (2.25).

In the following lemma, part (1) was proved by Chou-Wang [5, p. 1037]. We include here a slightly different proof (which provides the sharp constant $\theta=k / n$ ). It is part (2) that enables us to improve the estimates in [5] so that upper bound of $\lambda_{1}$ is independent of $\inf _{M} f$.

Lemma 2.2. Let $k$ be an integer between 1 and $n$. Suppose that $\mu=\left(\mu_{1}, \ldots, \mu_{n}\right) \in \Gamma_{k}$ with $\mu_{1} \geq \mu_{2} \geq \cdots \geq \mu_{n}$.

(1) We have

$$
\mu_{1} \sigma_{k-1}(\mu \mid 1) \geq \frac{k}{n} \sigma_{k}(\mu),
$$

where the quality holds if and only if $\mu_{1}=\mu_{2}=\cdots=\mu_{n}$.

(2) If there is a constant $B>0$ such that

$$
\sigma_{k}^{\frac{1}{k}-1}(\mu) \sigma_{k-1}(\mu) \leq B,
$$

then

$$
\sigma_{k}^{\frac{1}{k}-1}(\mu) \sigma_{k-1}(\mu \mid 1) \geq \frac{c(n, k)}{B^{k-1}},
$$

where $c(n, k)>0$ is a constant depending only on $n$ and $k$.

Proof. For $(1)$, since $\sigma_{k}(\mu)=\sigma_{k}(\mu \mid 1)+\mu_{1} \sigma_{k-1}(\mu \mid 1)$, it is equivalent to show that

$$
\mu_{1} \sigma_{k-1}(\mu \mid 1) \geq \frac{k}{n-k} \sigma_{k}(\mu \mid 1) .
$$

Note that

$$
\mu_{1} \geq \frac{\sigma_{1}(\mu \mid 1)}{n-1} .
$$

Next, we recall the generalized Newton-Maclaurin's inequality

$$
\frac{\sigma_{1}(\mu \mid 1)}{n-1} \geq \frac{k \sigma_{k}(\mu \mid 1)}{(n-k) \sigma_{k-1}(\mu \mid 1)},
$$

where the equality holds if and only if $\mu_{1}=\cdots=\mu_{n}$. Since $\sigma_{k-1}(\mu \mid 1)>0$, combining (2.36) and (2.37) yields (2.35), where the equality holds if and only if $\mu_{1}=\cdots=\mu_{n}$.

For (2), applying the generalized Newton-Maclaurin's inequality

$$
\left[\frac{\sigma_{k}(\mu) /\left(\begin{array}{l}
n \\
k
\end{array}\right)}{\sigma_{1}(\mu) / n}\right]^{\frac{1}{k-1}} \geq \frac{\sigma_{k}(\mu) /\left(\begin{array}{l}
n \\
k
\end{array}\right)}{\sigma_{k-1}(\mu) /\left(\begin{array}{c}
n \\
k-1
\end{array}\right)}
$$

and (2.33) yield that

$$
\frac{\sigma_{k}(\mu)^{\frac{1}{k}}}{\sigma_{1}(\mu)} \geq \frac{\left(\begin{array}{l}
n \\
k
\end{array}\right)}{n}\left(\frac{k}{n-k+1}\right)^{k-1} \frac{1}{B^{k-1}} .
$$


On the other hand, we have by part (1),

$$
\sigma_{k}^{\frac{1}{k}-1}(\mu) \sigma_{k-1}(\mu \mid 1) \geq \frac{k}{n} \frac{\sigma_{k}(\mu)^{\frac{1}{k}}}{\mu_{1}}>\frac{k}{n} \frac{\sigma_{k}(\mu)^{\frac{1}{k}}}{\sigma_{1}(\mu)} .
$$

This together with (2.38) imply (2.34), with

$$
c(n, k)=\left(\begin{array}{l}
n \\
k
\end{array}\right) \frac{k}{n^{2}}\left(\frac{k}{n-k+1}\right)^{k-1} .
$$

It remains to prove the claim. We will use the following elementary inequality:

Proposition 2.3. Let $a$ and $b$ be two elements in $\mathbb{C}^{n}$. For any $0<\varepsilon<1$, the following inequality holds:

$$
|a+b|^{2} \geq \varepsilon|a|^{2}-\frac{\varepsilon}{1-\varepsilon}|b|^{2} .
$$

Proof. Note that

$$
|a+b|^{2}=|a|^{2}+|b|^{2}+a \bar{b}+b \bar{a} \geq|a|^{2}+|b|^{2}-2|a||b| .
$$

On the other hand, we have

$$
2|a||b| \leq(1-\varepsilon)|a|^{2}+\frac{1}{1-\varepsilon}|b|^{2} .
$$

The result then follows immediately.

Let us now proceed to prove the claim: In view of (2.14), we have

$$
\begin{aligned}
& \varphi^{\prime \prime} \sum_{i \in I} F^{i \bar{i}}\left|u_{i} u_{i \bar{i}}+\sum_{p=1}^{n} u_{p i} u_{\bar{p}}\right|^{2}=2 \sum_{i \in I} F^{i \bar{i}}\left|\frac{u_{1 \overline{1} i}}{1+u_{1 \overline{1}}}+\psi^{\prime} u_{i}\right|^{2} \\
& \geq 2 \delta \sum_{i \in I} \frac{F^{i \bar{i}}\left|u_{1 \overline{1} i}\right|^{2}}{\left(1+u_{1 \overline{1}}\right)^{2}}-\frac{2 \delta\left(\psi^{\prime}\right)^{2}}{1-\delta} \sum_{i \in I} F^{i \bar{i}}\left|u_{i}\right|^{2}, \quad \text { (by Proposition 2.3). }
\end{aligned}
$$

Recall that we set $\delta=(1+2 A)^{-1}$. By virtue of (2.12), we obtain that

$$
\frac{2\left(\psi^{\prime}\right)^{2} \delta}{1-\delta} \sum_{i \in I}^{n} F^{i \bar{i}}\left|u_{i}\right|^{2} \leq \psi^{\prime \prime} \sum_{i \in I} F^{i \bar{i}}\left|u_{i}\right|^{2} .
$$

Thus,

$$
\begin{aligned}
& -\sum_{i, j, p, q=1}^{n} \frac{F^{i \bar{j}, p \bar{q}} u_{i \bar{j} 1} u_{p \bar{q} \overline{1}}}{1+u_{1 \overline{1}}}-\sum_{i \in I} \frac{F^{i \bar{i}}\left|u_{1 \overline{1} i}\right|^{2}}{\left(1+u_{1 \overline{1}}\right)^{2}}+\psi^{\prime \prime} \sum_{i=1}^{n} F^{i \bar{i}}\left|u_{i}\right|^{2} \\
& +\varphi^{\prime \prime} \sum_{i \in I} F^{i \bar{i}}\left|u_{i} u_{i \bar{i}}+\sum_{p=1}^{n} u_{p i} u_{\bar{p}}\right|^{2} \\
& \geq-\sum_{i, j, p, q=1}^{n} \frac{F^{i \bar{j}, p \bar{q}} u_{i \bar{j} 1} u_{p \bar{q} \overline{1}}}{1+u_{1 \overline{1}}}-(1-2 \delta) \sum_{i \in I} \frac{F^{i \bar{i}}\left|u_{1 \overline{1} i}\right|^{2}}{\left(1+u_{1 \overline{1}}\right)^{2}}
\end{aligned}
$$


To prove this claim, it is sufficient to show that

$$
-\sum_{i, j, p, q=1}^{n} \frac{F^{i \bar{j}, p \bar{q}} u_{i \bar{j} 1} u_{p \bar{q} \overline{1}}}{1+u_{1 \overline{1}}}-(1-2 \delta) \sum_{i \in I} \frac{F^{i \bar{i}}\left|u_{1 \overline{1} i}\right|^{2}}{\left(1+u_{1 \overline{1}}\right)^{2}} \geq 0 .
$$

Notice that, by (2.16),

$$
\begin{aligned}
& -\sum_{i, j, p, q=1}^{n} \frac{F^{i \bar{j}, p \bar{q}} u_{i \bar{j} 1} u_{p \bar{q} \overline{1}}}{1+u_{1 \overline{1}}} \\
& \geq-\sum_{i, p=1}^{n} \frac{F^{i \bar{i}, p \bar{p}} u_{i \overline{1}} u_{p \bar{p} \overline{1}}}{1+u_{1 \overline{1}}}-\sum_{i=2}^{n} \frac{F^{i \overline{1}, 1 \bar{i}}\left|u_{1 \overline{1} i}\right|^{2}}{1+u_{1 \overline{1}}}-\sum_{i=2}^{n} \frac{F^{1 \bar{i}, i \overline{1}}\left|u_{\bar{i} 11}\right|^{2}}{1+u_{1 \overline{1}}} \\
& \geq-\sum_{i=2}^{n} \frac{F^{i \overline{1}, 1 \bar{i}}\left|u_{1 \overline{1}}\right|^{2}}{1+u_{1 \overline{1}}}-\sum_{i=2}^{n} \frac{F^{1 \bar{i}, i \overline{1}}\left|u_{\bar{i} 11}\right|^{2}}{1+u_{1 \overline{1}}} \quad \text { (by concavity of } F \text { ) } \\
& \geq-\sum_{i \in I} \frac{F^{i \overline{1}, 1 \bar{i}}\left|u_{1 \overline{1} i}\right|^{2}}{1+u_{1 \overline{1}}} .
\end{aligned}
$$

To show (2.40), it suffices to prove that

$$
-F^{i \overline{1}, 1 \bar{i}} \geq(1-2 \delta) \frac{F^{i \bar{i}}}{1+u_{1 \overline{1}}}, \quad \text { for each } i \in I .
$$

This is equivalent to prove that,

$$
\lambda_{1} \sigma_{k-2}(\lambda \mid i 1) \geq(1-2 \delta) \sigma_{k-1}(\lambda \mid i), \quad \text { for each } i \in I .
$$

Since $\lambda_{1}>\lambda_{i}$ for each $i \in I$, we can write

$$
\sigma_{k-2}(\lambda \mid i 1)=\frac{\sigma_{k-1}(\lambda \mid i)-\sigma_{k-1}(\lambda \mid 1)}{\lambda_{1}-\lambda_{i}} .
$$

Thus, (2.41) is in turn equivalent to that

$$
\left(2 \delta+(1-2 \delta) \frac{\lambda_{i}}{\lambda_{1}}\right) \sigma_{k-1}(\lambda \mid i) \geq \sigma_{k-1}(\lambda \mid 1), \quad \text { for each } i \in I .
$$

Let us now proceed to prove (2.42): For each $i \in I$, we have

$$
\sigma_{k-1}(\lambda \mid i)>\delta^{-1} \sigma_{k-1}(\lambda \mid 1) .
$$

Then,

$$
\left(2 \delta+(1-2 \delta) \frac{\lambda_{i}}{\lambda_{1}}\right) \sigma_{k-1}(\lambda \mid i) \geq\left(2+(1-2 \delta) \frac{\lambda_{i}}{\delta \lambda_{1}}\right) \sigma_{k-1}(\lambda \mid 1) .
$$

We have assumed in this second case that $\lambda_{n} \geq-\delta \lambda_{1}$. In view of (2.13),

$$
\lambda_{i} \geq-\delta \lambda_{1}, \quad \text { for all } i \in I \text {. }
$$

Therefore,

$$
\left(2 \delta+(1-2 \delta) \frac{\lambda_{i}}{\lambda_{1}}\right) \sigma_{k-1}(\lambda \mid i) \geq(1+2 \delta) \sigma_{k-1}(\lambda \mid 1) .
$$

This implies (2.42). Hence, the claim is proved, and this completes the estimates in Case 2. 
Combining the two cases, we obtain that

$$
\lambda_{1} \leq C K=C\left(\sup _{M}|\nabla u|^{2}+1\right),
$$

Here and in the rest of this section, we denote by $C$ a generic positive constant depending only on $C_{0}, n, \sup |u|, \sup f^{1 / k}$, and $\sup \left|\nabla\left(f^{1 / k}\right)\right|^{2}$, and $\sup \left|d d^{c}\left(f^{1 / k}\right)\right|$. The estimate (2.43) would imply that

$$
\left|d d^{c} u\right|_{\omega}=\left[g^{i \bar{q}} g^{p \bar{j}} u_{i \bar{j}} u_{p \bar{q}}\right]^{1 / 2} \leq C\left(\sup _{M}|\nabla u|^{2}+1\right) .
$$

Indeed, since $\omega_{u} \in \Gamma_{k}$, we have $\sigma_{1}\left(\omega_{u}\right)>0$, and hence, at $x_{0}$,

$$
-\lambda_{n}<\lambda_{1}+\cdots+\lambda_{n-1} \leq(n-1) \lambda_{1} \leq C K \text {. }
$$

Then,

$$
\begin{aligned}
\left|\omega+d d^{c} u\right|_{\omega} & \leq n^{2} W\left(x_{0}, \xi_{0}\right) \exp (-\varphi-\psi) \\
& \leq n^{2} \lambda_{1} \exp (\sup \varphi-\inf \varphi) \exp (\sup \psi-\inf \psi) \\
& \leq \sqrt{2} 3^{A} n^{2} \lambda_{1}, \quad(\text { by }(2.7) \text { and }(2.10)), \\
& \leq C K, \quad(\text { by }(2.43)) .
\end{aligned}
$$

Therefore, Theorem 1.1 is proved, by virtue of Lemma 2.1 .

\section{Acknowledgment}

Part of the work was done while the second named author was visiting the Ohio State University in November 2008, he would like to thank their warm hospitality. The third named author would like to thank Qun Li for helpful discussions; he would also like to thank the support of OSU Newark campus. The first named author would like to thank Professor G. Tian and Professor D. H. Phong for their constant encouragement and help.

\section{References}

[1] Z. Błocki, Weak solutions to the complex Hessian equation, Ann. Inst. Fourier (Grenoble), 55 (2005), no. 5, 1735-1756.

[2] L. Caffarelli, L. Nirenberg and J. Spruck, The Dirichlet problem for nonlinear second order elliptic equations, III: Functions of the eigenvalues of the Hessian, Acta Math., 155 (1985), 261-301.

[3] L. Caffarelli, L. Nirenberg and J. Spruck, Nonlinear second order elliptic equations IV: Starshaped compact Weigarten hypersurfaces, Current topics in partial differential equations, Y. Ohya, K. Kasahara and N.Shimakura (eds), Kinokunize, Tokyo, 1985, 1-26.

[4] S. Y. Chang, M. Gursky and P. Yang, An equation of Monge-Ampre type in conformal geometry, and four-manifolds of positive Ricci curvature, Ann. of Math., 155 (2002), 709-787.

[5] K. S. Chou and X. J. Wang, A variation theory of the Hessian equation, Comm. Pure Appl. Math., 54 (2001), 1029-1064.

[6] P. Guan and X. N. Ma, The Christoffel-Minkowski problem I: convexity of solutions of a Hessian equations, Invent. Math., 151 (2003), 553-577.

[7] P. Guan and G. Wang, Local estimates for a class of fully nonlinear equations arising from conformal geometry, Int. Math. Res. Not., 23 (2003), 1413-1432.

[8] Z. Hou, Complex Hessian equation on Kähler manfolds, Int. Math. Res. Not. 2009, no. 16, 3098-3111. 
[9] N. M. Ivochkina, Solutions of the Dirichlet problem for certain equations of Monge-Ampère type (in Russian), Mat. Sb., 128 (1985), 403-415: English translation in Math. USSR Sb., 56 (1987).

[10] S. Y. Li, On the Dirichlet problems for symmetric function equations of the eigenvalues of the complex Hessian, Asian J. Math., 8 (2004), no. 1, 87-106.

[11] Y. Y. Li, Some existence results for fully nonlinear elliptic equations of Monge-Ampre type, Comm. Pure Appl. Math., 43 (1990), no. 2, 233-271.

[12] A. V. Pogorelov, The Minkowski Multidimensional Problem, John Wiley, 1978.

[13] J. Urbas, Hessian Equations on compact Riemannian Manifolds, Nonlinear Problems in Mathematical Physics and Related Topics II, Edited by Birman et al., Kluwer Academic/Plenum Publishers, New York, 2002, 369-377.

[14] X. J. Wang, The k-Hessian equation, Lecture Notes in Mathematics, Springer Berlin/Heidelberg, Volume 1977, 2009, 177-252.

[15] S. T. Yau, On the Ricci curvature of a compact Kähler manifold and the complex MongeAmpère equation I, Comm. Pure Appl. Math., 31 (1978), 339-411.

Department of Mathematics, Columbia University, New York, NY 10027, USA

E-mail address: hou@math.columbia.edu

Department of Mathematics, University of Science and Technology of China, Hefei, 230026, Anhui Province, CHINA

E-mail address: xinan@ustc.edu.cn

Department of Mathematics, The Ohio State University, 1179 University Drive, Newark, OH 43055, USA

E-mail address: dwu@math.ohio-state.edu 